\title{
L-BAND H POLARIZED MICROWAVE EMISSION DURING THE CORN GROWTH CYCLE
}

\author{
A.T. Joseph ${ }^{1}$, R. van der Velde ${ }^{2}$, P.E. O'Neill ${ }^{1}$, E. Kim ${ }^{1}$, R.H. Lang ${ }^{3}$ and T. Gish ${ }^{4}$ \\ 'Faculty of Geo-Information Science and Earth Observation (ITC), University of Twente, Hengelosestraat \\ 99, P.O. Box 6, 7500 AA, Enschede, The Netherlands, e-mail: velde@itc.nl, b_su@itc.nl \\ ${ }^{2}$ Hydrological Sciences Branch/614.3, Hydrospheric and Biospheric Sciences Laboratory, NASA/Goddard \\ Space Flight Center, Greenbelt, MD 20771 USA, Tel: 301-614-5804, Fax: 301 -614-5808, e-mail: \\ Alicia.T.Joseph@nasa.gov, Peggy.E.ONeill@nasa.gov
}

${ }^{3}$ Dept. of Electrical and Computer Engineering, George Washington University, Washington, DC 20052 USA, Tel: 202-994-6199, Fax: 202-994-0227, e-mail: lang@gwu.edu

${ }^{4}$ Hydrology \& Remote Sensing Lab, USDA-ARS, Beltsville, MD 20705 USA, Tel: 301-504-8378, Fax: 301504-8931, e-mail: tgish@hydrolab.arsusda.gov

Hourly L-band $(1.4 \mathrm{GHz})$ horizontally $(\mathrm{H})$ polarized brightness temperatures $\left(T_{\mathrm{B}}\right.$ 's) measured during five episodes (more than two days of continuous measurements) of the 2002 corn growth cycle are analyzed. These $T_{\mathrm{B}}$ measurements were acquired as a part of a combined active/passive microwave field campaign, and were obtained at five incidence and three azimuth angles relative to the row direction. In support of this microwave data collection, intensive ground sampling took place once a week. Moreover, the interpretation of the hourly $T_{\mathrm{B}}$ 's could also rely on the data obtained using the various automated instruments installed in the same field.

In this paper, the soil moisture and temperature measured at fixed time intervals have been employed as input for the tau-omega model to reproduce the hourly $T_{\mathrm{B}}$. Through the calibration of the vegetation and surface roughness parameterizations, the impact of the vegetation morphological changes on the microwave emission and the dependence of the soil surface roughness parameter, $h_{\mathrm{r}}$, on soil moisture are investigated. This analysis demonstrates that the $b$ parameter, appearing in the representation of the canopy opacity, has an angular dependence that varies throughout the growing period and also that the parameter $h_{\mathrm{r}}$ increases as the soil dries in a portion of the dry-down cycle. The angular dependence of the $b$ parameter imposes the largest uncertainty on $T_{\mathrm{B}}$ simulations near senescence as the response of $b$ to the incidence is also affected by the crop row orientation. On the other hand, the incorporation of a soil moisture dependent $h_{\mathrm{r}}$ parameterization was responsible for the largest error reduction of $T_{\mathrm{B}}$ simulations in the early growth cycle. 